\title{
Parametric excitation of plasma oscillations in Josephson Junctions
}

\author{
Pedersen, Niels Falsig; Samuelsen, Mogens Rugholm; Særmark, Knud
}

Published in:

Journal of Applied Physics

Link to article, DOI:

$10.1063 / 1.1662102$

Publication date:

1973

Document Version

Publisher's PDF, also known as Version of record

Link back to DTU Orbit

Citation (APA):

Pedersen, N. F., Samuelsen, M. R., \& Særmark, K. (1973). Parametric excitation of plasma oscillations in Josephson Junctions. Journal of Applied Physics, 44(11), 5120-5124. https://doi.org/10.1063/1.1662102

\section{General rights}

Copyright and moral rights for the publications made accessible in the public portal are retained by the authors and/or other copyright owners and it is a condition of accessing publications that users recognise and abide by the legal requirements associated with these rights.

- Users may download and print one copy of any publication from the public portal for the purpose of private study or research.

- You may not further distribute the material or use it for any profit-making activity or commercial gain

- You may freely distribute the URL identifying the publication in the public portal

If you believe that this document breaches copyright please contact us providing details, and we will remove access to the work immediately and investigate your claim. 


\title{
Parametric excitation of plasma oscillations in Josephson junctions
}

\author{
N. F. Pedersen, M. R. Samuelsen, and K. Saermark \\ Physics Laboratory I, The Technical University of Denmark, DK-2800 Lyngby, Denmark \\ (Received 18 June 1973)

\begin{abstract}
A theory is presented for parametric excitation of plasma oscillations in a Josephson junction biased in the zero voltage mode. A threshold curve for the onset of the parametric excitation is deduced via the stability properties of a Mathieu differential equation obtained by a self-consistent linearization procedure. The important parameters turn out to be the plasma frequency and the circuit losses. The results of the calculation are compared with the experiments performed on a Josephson junction analog and good agreement is obtained. It is suggested that the effect should be observable in tunnel junctions, but perhaps less likely in point contacts.
\end{abstract}

\section{INTRODUCTION}

The possibility of using a Josephson junction as a parametric amplifier has been investigated previously, theoretically ${ }^{1,2}$ as well as experimentally.$^{2-4}$ In these references the Josephson frequency $\omega_{0}=2 \mathrm{eV}_{0} / \hbar$ is used as the pump frequency and an external cavity is used $\mathrm{d}^{2-4}$ as the input circuit. Here $e$ is the electron charge, $2 \pi \hbar$ is Planck's constant, and $V_{0}$ is the voltage across the junction. The present paper describes a scheme for the parametric excitation of plasma oscillations in Josephson junctions. In Sec. II the junction model is presented, and the relevant circuit equation is set up. The junction is operated in the zero-voltage mode, and the junction plasma frequency acts as the input resonance frequency. Finally, the pump is external. It is shown that the nonlinear circuit equation subject to certain restrictions may be linearized, the ensuing equation being a Mathieu equation. As is well known, ${ }^{5}$ this equation has solutions which may be interpreted in terms of parametric excitation. In the present case the discus sion results in the deduction of a threshold curve for the onset of parametric excitation of plasma oscillations in the junction. In Sec. III the results obtained are illustrated by experiments performed on a Josephson-junction analog previously described. ${ }^{6}$ A quantitative comparison of the theory with the results of the analog experiments show a reasonably good agreement. Finally, in Sec. IV the possibility of observing the effect in a real Josephson junction is briefly discussed.

\section{THEORY}

The basic equations are the usual Josephson equations:

$$
\begin{gathered}
I_{J}=I_{0} \sin \phi, \\
V=\hbar \dot{\phi} / 2 e,
\end{gathered}
$$

where $I_{0}$ is the maximum dc Josephson current, $\phi$ the phase difference between the two superconductors of the junction, and $V$ the voltage across the junction. Furthermore, it is assumed that the Stewart-McCumber model $^{7,8}$ shown in Fig. 1 is applicable as a model for a Josephson tunnel junction or for a point contact. The resistor $R$ represents the losses in the junction, and the capacitor $C$ represents the capacitance between the two superconductors. Finally, the junction is regarded as current driven, the applied current consisting of a dc current $I_{\mathrm{dc}}$ and a rf current $I_{\mathrm{rr}} \cos (\omega t)$.

Now, assuming that the total current through the junction is equal to the applied current, one obtains the nonlinear equation

$$
I_{\mathrm{dc}}+I_{\mathrm{rf}} \cos (\omega t)=\frac{\hbar C}{2 e}\left(\ddot{\phi}+\frac{1}{R C} \dot{\phi}+\frac{2 e I_{0}}{\hbar C} \sin \phi\right) .
$$

In order to linearize this equation it is assumed that $\phi$ may be written in the form

$$
\phi=\phi_{0}+\phi_{1} \cos (\omega t-\theta)+\delta \phi,
$$

where $\phi_{0}, \phi_{1}$, and $\theta$ are constants and where $\delta \phi$ satisfies the condition

$$
\delta \phi \ll 1 \text {. }
$$

Inserting Eq. (2) in Eq. (1) and using condition (3) one obtains

$$
\begin{aligned}
\delta \ddot{\phi}+ & \frac{1}{R C} \delta \dot{\phi}+\frac{2 e I_{0}}{\hbar C} \cos \left[\phi_{0}+\phi_{1} \cos (\omega t-\theta)\right] \delta \phi \\
= & \frac{2 e}{\hbar C} I_{\mathrm{dc}}+\frac{2 e}{\hbar C} I_{\mathrm{rf}} \cos (\omega t)+\phi_{1} \omega^{2} \cos (\omega t-\theta) \\
& +\phi_{1} \omega \sin (\omega t-\theta)-\frac{2 e I_{0}}{\hbar C} \sin \left[\phi_{0}+\phi_{1} \cos (\omega t-\theta)\right] .
\end{aligned}
$$

It may now be seen that the linearization procedure can be carried through in a self-consistent way if the following additional conditions can be fulfilled: (a) The constants $\phi_{0}, \phi_{1}$, and $\theta$ are chosen such that there are no dc terms and no ac terms at the frequency $\omega$ present on the right-hand side of Eq. (4). (b) The remaining driving terms on the right-hand side of Eq. (4) are small in a sense to be specified below.

By using a Fourier-Bessel expansion of the last term on the right-hand side of Eq. (4), it may be shown that the following choice of the constant $\phi_{0}, \phi_{1}$, and $\theta$ will secure that there are no dc terms and no ac terms at the frequency $\omega$ left on the right-hand side of Eq. (4) as required by condition (a):

$$
I_{\mathrm{dc}}=I_{0} \sin \phi_{0} J_{0}\left(\phi_{1}\right),
$$

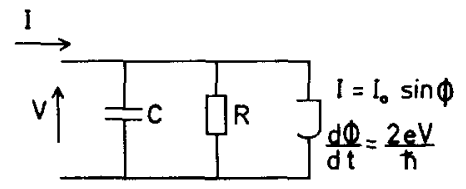

FIG. 1. Josephson-junction model. 


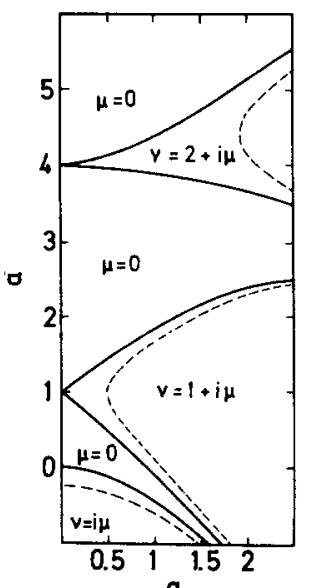

FIG. 2. Stability diagram for the Mathieu equation. (From Ref. 10.) Dashed curves: $\mu=0.5$.

$$
\begin{aligned}
& I_{\mathrm{rt}}=\phi_{1} \frac{\hbar C}{2 e}\left[\left(\frac{2 J_{1}\left(\phi_{1}\right)}{\phi_{1}} \omega_{p}^{2}-\omega^{2}\right)^{2}+\left(\frac{\omega}{R C}\right)^{2}\right]^{1 / 2}, \\
& \tan \theta=\frac{\omega / R C}{\left[2 J_{1}\left(\phi_{1}\right) / \phi_{1}\right] \omega_{p}^{2}-\omega^{2}} .
\end{aligned}
$$

Here, $J_{n}\left(\phi_{1}\right)$ is the usual Bessel function of order $n$ and $\omega_{p}$ is the Josephson plasma frequency given by

$$
\omega_{b}^{2}=\left(2 e I_{0} / \hbar C\right) \cos \phi_{0} .
$$

With this choice of the constants $\phi_{0}, \phi_{1}$, and $\theta, \mathrm{Eq}$. (4) takes the form

$$
\begin{aligned}
\delta \ddot{\phi}+ & \frac{1}{R C} \delta \dot{\phi}+\frac{2 e I_{0}}{\hbar C} \cos \left[\phi_{0}+\phi_{1} \cos (\omega t-\theta)\right] \delta \phi \\
= & \omega_{p}^{2}\left\{\tan \left(\phi_{0}\right) 2 J_{2}\left(\phi_{1}\right) \cos [2(\omega t-\theta)]\right. \\
& \left.+2 J_{3}\left(\phi_{1}\right) \cos [3(\omega t-\theta)]-\cdots\right\} .
\end{aligned}
$$

Condition (b) amounts to requiring that $2 J_{2}\left(\phi_{1}\right) \ll 1$, $2 J_{3}\left(\phi_{1}\right) \ll 1$, etc., which yields the condition

$$
\phi_{1}^{2} \ll 1 \text {. }
$$

Using, finally, a Fourier-Bessel expansion of the cos term on the left-hand side of Eq. (6), one ends up with the equation

$$
\begin{aligned}
\delta \ddot{\phi}+ & \frac{1}{R C} \delta \dot{\phi}+\omega_{p}^{2}\left[J_{0}\left(\phi_{1}\right)-\tan \left(\phi_{0}\right) 2 J_{1}\left(\phi_{1}\right) \cos (\omega t-\theta)\right] \delta \phi \\
& =\omega_{p}^{2} \tan \left(\phi_{0}\right) 2 J_{2}\left(\phi_{1}\right) \cos [2(\omega t-\theta)],
\end{aligned}
$$

where $\theta$ is determined by Eq. (5) and where the Bessel functions can be replaced by the expansions

$$
J_{0}\left(\phi_{1}\right) \approx 1-\left(\frac{1}{2} \phi_{1}\right)^{2}, \quad 2 J_{1}\left(\phi_{1}\right) \approx \phi_{1}, \quad 2 J_{2}\left(\phi_{1}\right) \approx\left(\frac{1}{2} \phi_{1}\right)^{2} .
$$

The complete solution to Eq. (8) is given as a sum of the complete solution to the corresponding homogeneous equation and a particular solution to Eq. (8). The latter is for small $\phi_{1}$ given as the solution to a forced damped harmonic oscillator:

$$
\begin{aligned}
\delta \phi_{\text {part }} & =\frac{\omega_{p}^{2} \tan \left(\phi_{0}\right)\left(\frac{1}{2} \phi_{1}\right)^{2}}{\left[\left(\omega_{p}^{2}-4 \omega^{2}\right)^{2}+(2 \omega / R C)^{2}\right]^{1 / 2}} \cos \left[2(\omega t-\theta)-\theta^{\prime}\right], \\
\tan \left(\theta^{\prime}\right) & =\frac{2 \omega / R C}{\omega_{p}^{2}-4 \omega^{2}} .
\end{aligned}
$$

The voltage corresponding to $\mathrm{Eq}$. (9) was the one measured in the plasma resonance experiments. ${ }^{9}$

In order to examine the complete solution to the homogeneous equation corresponding to $\mathrm{Eq}$. (8), the following substitution is made:

$$
\delta \phi=\exp (-t / 2 R C) \delta \phi^{\prime},
$$

which transforms the homogeneous part of Eq. (8) into

$$
\delta \ddot{\phi}^{\prime}+\left[\omega_{p}^{2}-(1 / 2 R C)^{2}-\phi_{1} \tan \left(\phi_{0}\right) \omega_{p}^{2} \cos (\omega t-\theta)\right] \delta \phi^{\prime}=0 \text {. }
$$

By comparison of Eq. (11) with one of the standard forms of the Mathieu equation, see Ref. 10,

$$
\frac{d^{2} y}{d z^{2}}+[a-2 q \cos (2 z)] y=0,
$$

it is seen that Eq. (11) is a Mathieu equation with the following choice of the parameters $a$ and $q$ :

$$
\begin{aligned}
& a=\frac{\left(2 \omega_{p}\right)^{2}-(1 / R C)^{2}}{\omega^{2}}=\left(\frac{2 \omega_{p}}{\omega}\right)^{2}\left[1-\left(\frac{1}{2 Q}\right)^{2}\right], \\
& 2 q=\left(\frac{2 \omega_{p}}{\omega}\right)^{2} \phi_{1} \tan \left(\phi_{0}\right)=\left(\frac{2 \omega_{p}}{\omega}\right)^{2} \alpha \tan \left(\phi_{0}\right),
\end{aligned}
$$

where $Q=\omega_{p} R C$ and, to be in accordance with the more common notation, $\alpha$ has been introduced as

$$
\alpha=2 e V_{\mathrm{rf}} / \hbar \omega=\phi_{1} \text {. }
$$

The complete solution to Eq. (11) is given by ${ }^{10}$

$$
\begin{aligned}
\delta \phi^{\prime}= & A \exp \left[i \nu\left(\frac{1}{2} \omega t\right)\right] P\left(\frac{1}{2} \omega t\right) \\
& +B \exp \left[-i \nu\left(\frac{1}{2} \omega t\right)\right] P\left(-\frac{1}{2} \omega t\right)
\end{aligned}
$$

where $A$ and $B$ a re arbitrary constants, $\nu=\nu_{\tau}+i \mu$ is the characteristic exponent, and $P(z)$ is a periodic function with period $\pi$, i.e., $P\left(\frac{1}{2} \omega t\right)$ is periodic in $(\omega t)$ with the period $2 \pi$. As a consequence of Eqs. (12) and (10) the complete solution to the homogeneous part of Eq. (8) is

$$
\begin{aligned}
\delta \phi= & A \exp \left[-\left(\omega_{p} / 2 Q+\frac{1}{2} \mu \omega\right) t\right] \exp \left[i \nu_{r}\left(\frac{1}{2} \omega t\right)\right] P\left(\frac{1}{2} \omega t\right) \\
& +B \exp \left[\left(-\omega_{p} / 2 Q+\frac{1}{2} \mu \omega\right) t\right] \exp \left(-i \nu_{r}\left(\frac{1}{2} \omega t\right)\right] P\left(\frac{1}{2} \omega t\right) .
\end{aligned}
$$

In the discussion of the complete solution Eq. (13), the dependence of the characteristic exponent $\nu$ on the parameters $a$ and $q$ enters in an essential way. In Fig. 2 a part of the stability diagram for the Mathieu equation is shown. In regions where $\mu=0 \mathrm{Eq}$. (11) has stable solutions for $\delta \phi^{\prime}$, and therefore one gets damped solutions for $\delta \phi$ as shown by Eq. (13). In regions where $\mu \neq 0$ one gets constant and integer values for $\nu_{r}$. In these regions $\exp \left[i \nu_{r}\left(\frac{1}{2} \omega t\right)\right] P\left(\frac{1}{2} \omega t\right)$ is periodic with the frequencies $\frac{1}{2}\left(2 n+\nu_{r}\right) \omega$ For even values of $\nu_{r}$ the corresponding frequencies are $\omega, 2 \omega, 3 \omega, \ldots$, whereas for odd values of $\nu_{r}$ the corresponding frequencies are $\omega / 2,3 \omega / 2,5 \omega / 2, \ldots$.

In the regions where $\mu \neq 0$ solution (13) for $\delta \phi$ may obviously become unstable. The first term is always exponentially damped. The second term, however, will be damped only if $Q<\left(2 \omega_{p} / \omega\right)(1 / 2 \mu)$. On the contrary, if $Q$ satisfies the inequality

$$
Q>\frac{2 \omega_{p}}{\omega} \frac{1}{2 \mu} \approx \frac{\sqrt{a}}{2 \mu},
$$




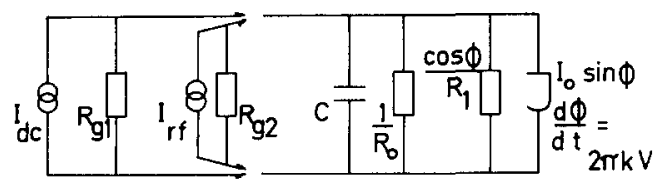

FIG. 3. Analog equivalent circuit; $R_{g 1}=10 \mathrm{k} \Omega, R_{g^{2}}=2.8 \mathrm{k} \Omega$. $1 / R_{\text {eff }}=1 / R_{g 1}+1 / R_{g 2}+1 / R_{0}-\left(1 / R_{1}\right) \cos \phi_{0}$.

the second term will be unstable in the sense that the amplitude will increase exponentially with time. The value of $\mu$ to be inserted in Eq. (14) is, of course, a function of the parameters $a$ and $q$. In Fig. 2 the dotted curves show this dependence for the case $\mu=0.5$.

Considering, now, the special case $\nu_{r}=1$, or $\omega \sim 2 \omega_{p}$, condition (14) may for small losses be written

$$
\frac{2 q}{a}=\alpha \tan \left(\phi_{0}\right)>2\left\{\left[1-\left(\frac{\omega}{2 \omega_{p}}\right)^{2}\right]^{2}+\frac{1}{Q^{2}}\left(\frac{\omega}{2 \omega_{p}}\right)^{2}\right\}^{1 / 2} \text {. }
$$

The preceding discussion may now be summarized as follows :

(a) If the conditions for the linearization procedure, i.e., Eqs. (3) and (7) are fulfilled, and if the values of the parameters $a$ and $q$ are such that one is in a region of the stability diagram for the Mathieu equation where condition (14) is violated, then the "Ansatz" in Eq. (2) with a small $\delta \phi$ given by Eq. (9) is a solution to the starting Eq. (1).

(b) If the conditions for the linearization procedure, i.e., Eqs. (3) and (7) are fulfilled, and if the values of the parameters $a$ and $q$ are such that one is in a region of the stability diagram for the Mathieu equation where condition (14) is satisfied them, even if one in the "Ansatz" in Eq. (2) starts out with $\delta \phi=0$, a fluctuation in $\phi$ will bring in an exponentially increasing part, i.e., the second term of Eq. (13), such that sooner or later condition (3) will be violated and the linearization procedure invalid. However, the instability condition Eq. (14), itself is still valid. One should note that condition (7) also can be written

$$
\alpha^{2} \ll 1 \text {. }
$$

Finally, from the character of the solution to the linearized equation, i.e., the behavior of $\exp \left(\frac{1}{2} i \nu_{r} \omega t\right) P\left(\frac{1}{2} \omega t\right)$, it follows that the frequency content of the actual solution will be either $\omega / 2,3 \omega / 2, \ldots$ or $2 \omega, 3 \omega, \ldots$. In particular, one notes that if $\omega \sim 2 \omega_{p}$, then the solution will contain a frequency component of $\omega / 2 \sim \omega_{p}$. However, the amplitude of the half-harmonics and harmonics, respectively, can only be found solving the full nonlinear equation (1).

\section{ANALOG EXPERIMENTS}

In order to illustrate the parametric excitation of plasma oscillations predicted in the foregoing section some experiments were performed using a Josephsonjunction analog. The analog, which basically is a phaselocked loop with an equivalent diagram as shown in Fig. 3 , has been described earlier. ${ }^{6}$ It has the following

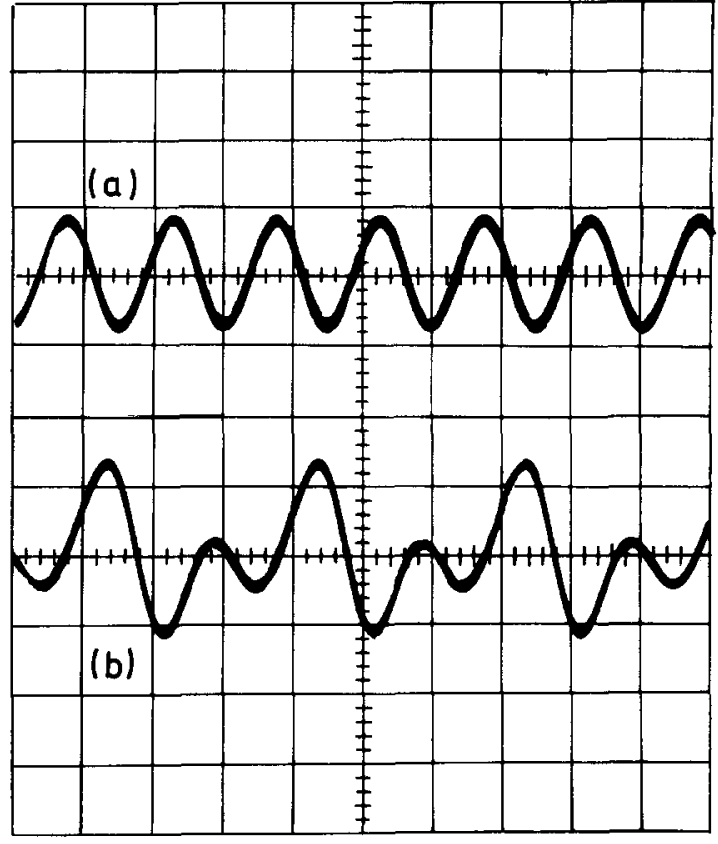

FIG. 4. Typical waveforms just below (a) and just above (b) $V_{\mathrm{rf}}^{\mathrm{crt}} \mathrm{t}$. Horizontal axis, $140 \mu \mathrm{s} /$ div; vertical axis, $10 \mathrm{mV} / \mathrm{div}$.

characteristics: $I_{0}=1 \mathrm{~mA}, k=2 e / \hbar=200 \mathrm{kHz} / \mathrm{V}$, cutoff frequency $f_{c}=100 \mathrm{kHz}$, and $R_{0}=500 \Omega$. The value of the $\cos \phi$ conductivity, which is inherent in the analog ${ }^{6}$ and which itself is the analog of the quasiparticle-pair interference term, ${ }^{9}$ depends on the frequency and can be adjusted by the reference signal to the mixer. ${ }^{6}$ For the particular experiment reported here, the following values apply: $C=100 \mathrm{nF}, \sin \left(\phi_{0}\right)=I_{\mathrm{dc}} / I_{0}=0.66$, and the "plasma frequency" $f_{b} \approx 14 \mathrm{kHz}$. The measurements were performed by applying an rf signal of frequency $f \sim 2 f_{p}$ and displaying the $\mathrm{rf}$ voltage across the junction on an oscilloscope. The $\mathrm{rf}$ power was gradually increased until, for a certain value of the rf voltage, $V_{\mathrm{rf}}^{\mathrm{cr}}$, , half of the applied frequency suddenly appeared in the voltage. Typical waveforms for the rf voltage just below and just above the threshold $\mathrm{rf}$ voltage $V_{\mathrm{rf}}^{\mathrm{er} i t}$ are shown in Fig. 4.

The threshold voltage depends on the frequency $f$. In the neighborhood of $f \sim 2 f_{p}$ it turned out to be possible to

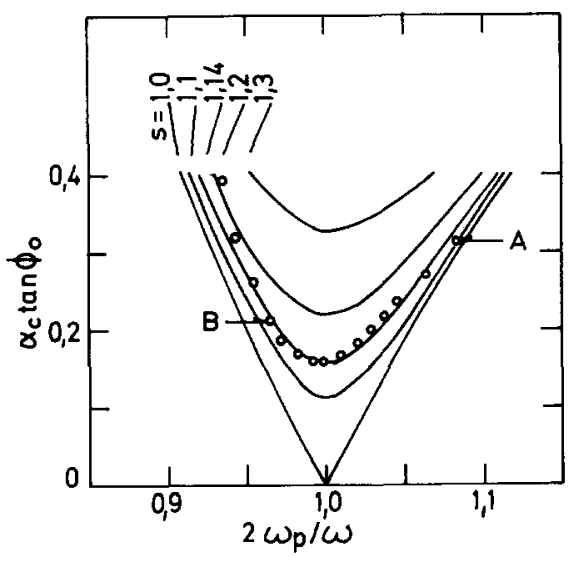

FIG. 5. $\alpha_{c} \tan \left(\phi_{0}\right)$ vs $2 \omega_{p} / \omega$. Full curves: stability curves for the Mathieu equation. Circles: analog experiments. 


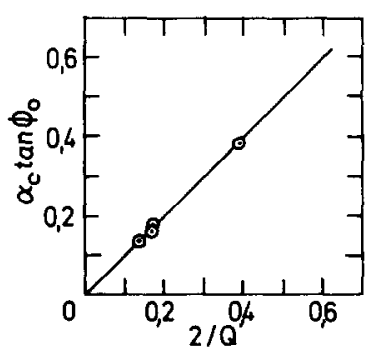

FIG. 6. $\alpha_{c} \tan \left(\phi_{0}\right)$ vs $2 / Q$. Straight line: Eq. (17). Circles: analog experiments.

follow this dependence in detail. Thus, Fig. 5 shows a plot of the quantity $\alpha_{c} \tan \left(\phi_{0}\right)=\left(k V_{\mathrm{rf}}^{\mathrm{cr} i t} / f\right) \tan \left(\phi_{0}\right)$ vs $2 \omega_{p} / \omega$ in the neighborhood of $f \approx 2 f_{p}$. Also shown are curves for constant values of $\mu$ or rather the parameter $s=\exp (\pi \mu)$, as taken from Ref. 10. By interpolation the curve corresponding to $s=1.14$ is found to give the best fit to the analog measurements. The minimum value for $\alpha_{c} \tan \left(\phi_{0}\right)$ occurs at $\omega=2 \omega_{p}$ and has the value 0.16 . According to the theory given in Sec. II, i.e., Eq. (15), this minimum value for the onset of parametric excitation is given by

$$
\left.\alpha_{c} \tan \left(\phi_{0}\right)\right|_{\omega=2 \omega_{p}}=2 / Q .
$$

Hence, we calculate $2 / Q$ by taking the total circuit losses into account and using the circuit constants given earlier. This gives

$$
2 / Q=2 / \omega_{p} C R_{\text {eff }}=0.17
$$

Thus, the agreement between the analog experiments and the theory is good. However, it should be noted that $R_{\text {eff }}$ depends also on the $\cos (\phi)$ conductivity of the analog and that this dependence gives a significant contribution to the above estimate of $2 / Q$. In the theory given in Sec. II, the quasiparticle-pair interference term has not been discussed.

From Fig. 5 one notes that deviations begin to occur for $\alpha_{c} \tan \left(\phi_{0}\right)>0.3$ corresponding to $\alpha_{c}^{2} \sim 0.1$. Thus, a possible explanation is that condition (i6) is beginning to be violated. It has further been demonstrated that the minimum threshold value varies as $2 / Q$ when extra losses are inserted in the analog, and that the $\tan \left(\phi_{0}\right)$ dependence is valid for a large range of $\phi_{0}$. The results are shown in Fig. 6. In particular, in the limit $I_{\mathrm{dc}} \rightarrow 0$ no parametric excitation was observed.

One might speculate whether or not a similar parametric excitation might be observed in other regions of the stability diagram for the Mathieu equation, i.e., at $\nu_{r}=2,3 \ldots$. In principle this should be the case, however, it would require very large values of $Q$.

By means of a spectrum analyzer the frequency content of the rf voltage was measured for a number of applied frequencies and for a number of power levels. Thus, in Fig. 5 the points $A$ and $B$ mark some of the frequencies at which this analysis was attempted. In general, a very complicated picture appears. Thus, at point $A$ the half-harmonic was $1 \mathrm{~dB}$, the $\frac{3}{2}$ harmonic, was $25 \mathrm{~dB}$, and the second harmonic was $40 \mathrm{~dB}$ below the applied signal. At point $B$ the corresponding numbers are 5,35 , and $35 \mathrm{~dB}$. Similarly one finds at point $\mathrm{C}$ $\left(2 \omega_{p} / \omega \sim 2\right) 11,13$, and $22 \mathrm{~dB}$. A somewhat different picture appears at points $D\left(2 \omega_{p} / \omega \sim 7\right)$ and $E\left(2 \omega_{p} / \omega \sim 3\right)$. Here the odd half-harmonics are very much suppressed, i.e., $\sim 50 \mathrm{~dB}$ below the applied signal, whereas there is an appreciable content of ordinary harmonics; e.g., at point $D$ one finds for the second, third, fourth, and fifth harmonics $7,1,4$, and $23 \mathrm{~dB}$, respectively, below the applied signal. The numbers quoted above should, of course, only be taken as an indication of the complicated frequency distribution and not as an attempt at a quantitative mapping.

One final point should be mentioned. In the analog experiments it was observed that a similar parametric excitation could be seen on the $n=1 \mathrm{rf}$-induced step. As the rf power was gradually increased the first halfharmonic appeared for a certain threshold value of the applied rf voltage. The analog did not change its dcbias point, but remained on the $n=1$ step. A preliminary analysis indicates that, in certain limiting situations, it may be possible to account theoretically for the excitation much along the lines set up in Sec. II.

\section{CONCLUDING REMARKS}

In Secs. I-III it was shown to be possible by means of a Mathieu equation to establish a threshold rf voltage below which a small-signal linearization of the circuit equation for a Josephson-junction model-the StewartMcCumber model-exposed to an external rf signal can be performed. Above the threshold a parametric excitation was shown to exist and was obse rved by means of experiments performed on a Josephson-junction analog described earlier. A good agreement between theory and experimental results were obtained. The question then remains: can the effect be expected to be observable in a real Josephson junction or, in other words, can the fundamental condition, Eq. (17), be satisfied for a real junction.

Considering first a Josephson tunnel junction, i.e., a crossed film junction, typical parameters may be taken as $I_{0}=4 \mathrm{~mA}, I_{\mathrm{dc}}=2 \mathrm{~mA}, C=1 \mathrm{nF}$, and $R_{N}=0.3 \Omega$ which yields $f_{p} \approx 18 \mathrm{GHz}$ and $\alpha_{c}=0.1$ for $f=36 \mathrm{GHz}$. In this estimate the effect of source impedance and quasiparticle-pair conductivity has been neglected. Considering next a point contact, typical parameters may be taken as $I_{0}=30 \mu \mathrm{A}, I_{\mathrm{dc}}=25 \mu \mathrm{A}, R=20 \Omega$, and $C=0.05$ $\mathrm{pF}$, which yields $f_{p} \approx 200 \mathrm{GHz}$ and $\alpha_{c}=1$ for $f \approx 400 \mathrm{GHz}$. More generally, the possibility of observing the effect in a point contact may be estimated from McCumber' ${ }^{8}$ $\beta$-vs- $\alpha$ curve ( $\alpha$ is the hysteresis parameter) since $\beta$ $=Q^{2} / \cos \left(\phi_{0}\right)$. The conclusion appears to be that it should be possible to observe the effect in tunnel junctions, whereas it appears less likely that the effect is observable with point contacts, although it is known experimentally that it is possible to couple such high frequencies to a point-contact junction. 


\section{ACKNOWLEDGMENT}

The authors are very grateful to C.K. Bak and Sidney Shapiro for many valuable discussions.

1P. Russer, Arch. Elek. Übertragung 23, 417 (1969) ${ }^{2}$ A. N. Vystaukin et al., Cornell Conference on High Frequency Generation and Amplification, 1971 (unpublished).
${ }^{3}$ H. Zimmer, Appl. Phys. Lett. 10, 193 (1967).

${ }^{4}$ H. Kanter and A. H. Silver, Appl. Phys. Lett. 19, 515 (1971). ${ }^{5}$ N. N. Bogolubow and J.A. Mitropolski, Asymptotische Methoden in der Theorie der nichtlinearen Schwingungen (Adademie-Verlag, Berlin, 1965), p. 225.

${ }^{6}$ C. K. Bak and N. F. Pedersen, Appl. Phys. Lett. 22, 149 (1973).

${ }^{7}$ W. C. Stewart, Appl. Phys. Lett. 12, 277 (1968).

${ }^{8}$ D. E. McCumber, J. Appl. Phys. 39, 3113 (1968).

${ }^{9}$ N. F. Pedersen, T.F. Finnegan, and D.N. Langenberg, Phys. Rev. B 6, 4151 (1972).

${ }^{10}$ M. Abramowitz and I.A. Stegun, Handbook of Mathematical Functions (Dover, New York, 1965). 